\title{
Tremor, CTCAE 5.0
}

National Cancer Institute

\section{Source}

National Cancer Institute. Tremor, CTCAE 5.0. NCI Thesaurus. Code C146780.

A disorder characterized by the uncontrolled shaking movement of the whole body or individual parts. 